\title{
Some Clinical and Metabolic Aspects of Essential Familial Hypercholesterolemia
}

\author{
Masamitsu SHIBA \\ Second Department of Internal Medicine, Faculty of Medicine, \\ University of Tokyo, Tokyo, Japan
}

In Japan there were few investigations regarding the essential familial hypercholesterolemia (EFH) apart from some case reports. In this article the author intended to elucidate some characteristic features in the clinical and especially biochemical aspects of EFH as compared with other types of hypercholesterolemia, that is, the "symptomatic" one commonly encountered in diabetes, nephrotic syndrome, obstructive jaundice etc., and the non-familial but most probably "essential" ") one without any causative factors of hypercholesterolemia as frequently seen in general atherosclerotic and/or hypertensive subjects, but occasionally of no clinical symptoms and signs.

The serum total cholesterol values obtained individually in the cases of above groups were indicated in Fig. 1, where those of the constituents of individual pedigrees of EFH were aligned in the same columns. Among these eleven pedigrees of EFH there were the twelve cases with various cardiovascular complications, mainly atherosclerotic, such as six cases of myocardial infarction, four of systemic hypertension, and each one of aortic arch syndrome and iliac artery thrombosis. Of these twelve cases the apparent xanthomatosis supervened in six,

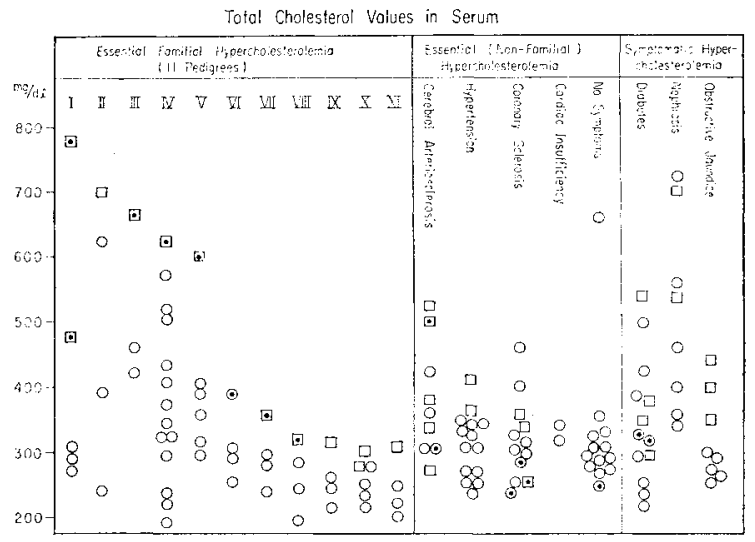

Fig. 1. Classification of the hypercholesterolemic subjects studied and the values of serum total cholesterol. Symbols are as follows: EFH (O) Without significant complications: $\square$ With cardiovascular complications), Cerebral arteriosclerosis (O Without evident apoplexy: $\square$ Apoplectic cases), Hypertension ( $O$ Simple hypertension: $\square$ With hypertensive and/or arteriosclerotic complications), Coronary sclerosis $(O$ Without evident myocardial infarction: $\square$ With myocardial infarction), Diabetes ( $O$ Without significant complications: With cardiovascular complications), Nephrotic syndrome ( $O$ Common nephritic syndrome: $\square$ Complicated by systemic lupus erythematosus), Obstructive jaundice $(O$ Various hepatitis: $\square$ Malignant liver tumor). Dotted cases are all those of xanthomatosis or xanthelasma $(\odot,[0)$. 
though one xanthomatous case of EFH seemed free from any other complications, and there were in general no definite correlations between the serum cholesterol value and the patient's sex, age, xanthomatosis and other complications. But it is frequently noted that the seriously complicated cases were of the highest cholesterol values in the constituents of same pedigrees of EFH. Of these serious cases the symptoms of angina pectoris and subsequent myocardial infarction occurred almost between their thirties and early forties, that is to say, there the relatively juvenile onset of such events may be rather characteristic, as noted in foreign countries.2) As for the hereditary mode the single dominant gen theory postulated by Muller and others ${ }^{3,4}$ ) seemed relevant also for the present pedigrees of EFH with or without xanthomatosis rather than the homo- and heterozygous gens theory by Wilkinson and others. ${ }^{5)}$

In connection with the enormous increase in the total cholesterol values as cited above there might be expected some abnormalities referring to the other lipid fractions as follows, here also particularly concerning EFH among others. However, the esterification of cholesterol that may be argued from the ratios of esterified cholesterol to total in serum seemed fairly normal in most cases including EFH except for some patients with obstructive jaundice, in which the actual involvement of the liver may cause the apparent reduction of that ester ratio (Fig. 2-a). Similarly the serum phospholipid in total was markedly increased, particularly in relation to the total
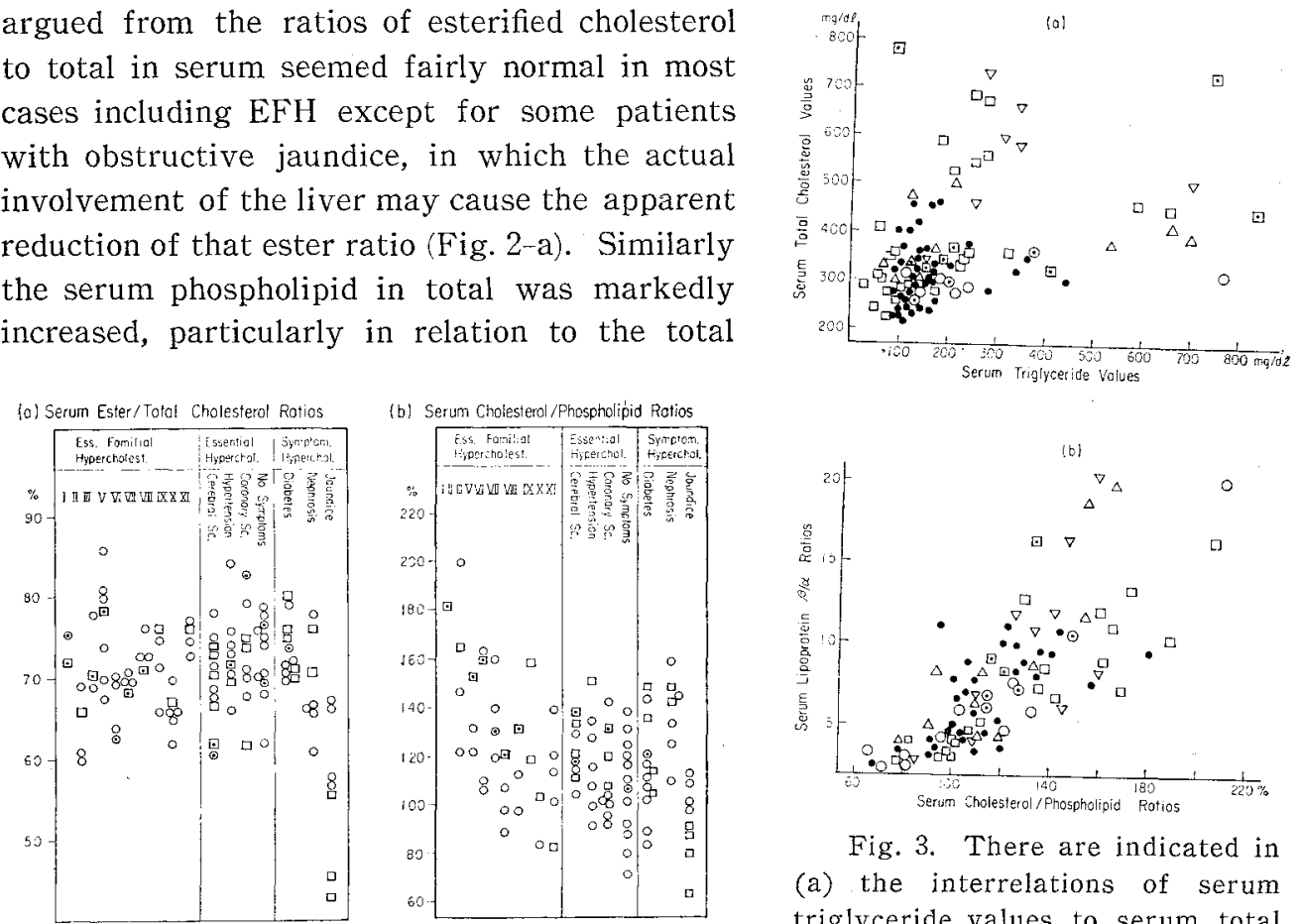

Fig. 2. In (a) indicated are the ratios of serum esterified-cholesterol to total cholesterol, and in (b) are the ratios of serum total cholesterol to total phospholipid. Classification of the cases and their individual symbols are the same as in Fig. 1.

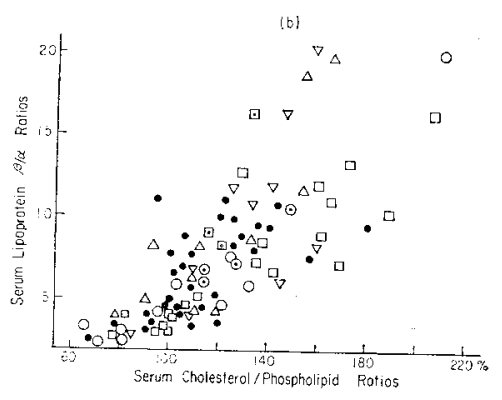

Fig. 3. There are indicated in (a) the interrelations of serum triglyceride values to serum total cholesterol values, and in (b) the relationship between the serum lipoprotein $\beta / \alpha$ ratios and the serum cholesterol/phospholipid ratios. Indicated symbols are the same as in Fig. 4. 
cholesterol more remarkably in the case of symptomatic hypercholesterolemia than the others. Thus there seemed some tendency that the ratio of total cholesterol to phospholipid $(c / p)$ in serum was somewhat higher in the case of essential hypercholesterolemia especially in EFH than the symptomatic ones (Fig. 2-b). Fairly same were the circumstances as for the $\beta / \alpha$ lipoprotein ratios determined electrophoretically, which consequently seemed in some parallel relationship with the $\mathrm{c} / \mathrm{p}$ ratios (Fig. 3-b). The serum triglyceride, however, was not in general characteristically elevated in $\mathrm{EFH}$, in which it remained mostly within $300 \mathrm{mg}$./ $\mathrm{dl}$. or so and only occasionally became near the value of total cholesterol (Fig. 3-a). At the same time there was seen generally no relation between the amounts of total cholesterol and those of triglyceride in serum.

The above observations concerning the various serum lipids may suggest some characteristics of EFH as compared with other types of hypercholesterolemia, which will be more clearly defined through the investigation of their fatty acid compositions in serum. This was performed in use of the gas-liquid chromatography ${ }^{6}$ after fractionation of serum lipids through the silicic acid column chromatography. ${ }^{7}$ ) Thus the percent compositions of usual fatty acids found for example in the cholesterol ester fraction of the individual cases were plotted against the tetra-axial reference systems as seen in Figs. $4-a$ and $-b$, so that the interrelations of the varying compositions in
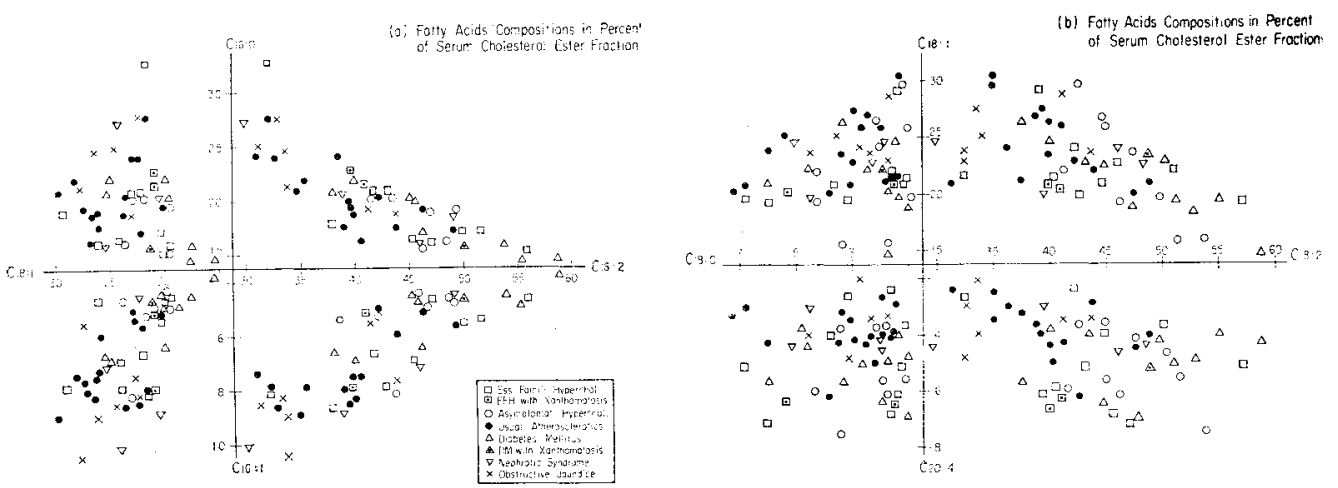

Fig. 4. In the tetra-axial reference systems (a) and (b), there are dotted the individual fatty acids compositions in percentage of serum cholesterol ester fraction, so that the interrelations of these fatty acids to each other are easily observed.

percentage of individual fatty acids may be easily observed. In the same figures there seemed some reverse relations as a whole between the "essential " fatty acids especially linoleate $(18: 2)$ and the other "saturated" and "monounsaturated" ones such as palmitate $(16: 0)$, palmitoleate $(16: 1)$ and oleate $(18: 1)$, while the latter two monounsaturated acids were rather positively correlated. In addition there it may be apparent that for the most part of the symptomatic hypercholesterolemia such as nephrotic syndrome and obstructive jaundice as well as the non-familial but likely 
essential hypercholesterolemia consisting mainly of usual atherosclerotics the apparent reduced percent compositions of linoleate as against the increased ones of oleate and palmitate were rather characteristic, while the fairly reverse interrelations of these fatty acids were true for the greater part of $\mathrm{EFH}$ and diabetes irrespective of their atherosclerotic complications. Similar circumstances concerning these main fatty acids respectively in the above-cited groups were mostly observed also in the serum triglyceride and phospholipid fractions, though in part not so evidently as in the cholesterol ester. But at least it may be generally concluded that in the serum fatty acid compositions the linoleate is not so significantly reduced but rather frequently increased in EFH, as particularly against the case of non-familial hypercholesterolemia commonly encountered in the usual atherosclerotics. This may be otherwise well compatible with some observations that the $\beta$-lipoproteins markedly increased in EFH as already described are essentially of relatively higher densities $\left(\mathrm{Sf}_{0-20}\right.$ classes) than those in the usual hypercholesterolemic atherosclerosis composed mainly of $\mathrm{Sf}_{20-400}$ other than $\mathrm{Sf}_{0-20}$ classes, ${ }^{8,9)}$ because the linoleate concentrations in various $\beta$-lipoprotein fractions are recently found much higher in the $\mathrm{Sf}_{0-20}$ than in the $\mathrm{Sf}_{20-400}$ classes particularly in the cholesterol ester fraction in serum among others. ${ }^{103}$

\section{References}

1) Zöllner, N.: "Erbliche Stoffwechselkrankheiten" edited by Linneweh, Urban, München, 1962.

2) Thannhauser, S. J.: “Lipidoses", Grune \& Stratton, New York \& London, 1958.

3) Muller, C.: Arch. Int. Med., 64: 675, 1939.

4) Wheeler, E. O.: Am. J. Med., $23: 653,1957$.

5) Wilkinson, C.F., Hand, F. A. and Fliegelman, M. T.: Ann. Int. Med., 29: 671, 1948.

6) Tanaka, K. and Takahashi, Y.: J. J.S. I. M., 50: 140, 1961.

7) Hirsch, J. and Ahrens, E. H.: J. B. C., $233: 311,1958$.

8) Gofman, J.W., Rubin, L., McGinley, J.P. and Jones, H. B.: Am. J. Med., 17: 514, 1954.

9) Guravich, J.L.: Am. J. Med., 26: 8, 1959.

10) Freeman, N.K., Lindgren, F. T. and Nichols, A.V.: In "Progress in the Chemistry of Fats and Other Lipids" edited by Holman, Lundberg and Malkin, Pergamon Press, Oxford, 1963 . 